\title{
Starspot migration in close binaries: A fast parameters evaluation from large sky surveys
}

\author{
B. Debski ${ }^{1}$ and S. Zola ${ }^{1,2}$ \\ ${ }^{1}$ Astronomical Observatory of the Jagiellonian University, \\ Orla 171, 30-244 Krakow, Poland \\ email: b.debski@oa.uj.edu.pl \\ ${ }^{2}$ Mt. Suhora Observatory, Pedagogical University, \\ ul. Podchorazych 2, 30-084 Krakow, Poland \\ email: szola@oa.uj.edu.pl
}

\begin{abstract}
We developed a method that allows to classify the light curves of eclipsing binaries of the W UMa type (EW) with respect to their intrinsic variability. The algorithm measures several features of light curves, such as the amplitude of the O'Connell effect, the separation and location of maxima brightness as well as depths of the minima in subsequent orbital periods. This method is capable of distinguishing systems with presumed magnetic activity present from these without it, as well as recognizing systems with starspots migration and those with other types of intrinsic variability manifestation. The classification is done in an automatic way without a time consuming, visual inspection of light curves.
\end{abstract}

Keywords. Stars, variable stars, classification

\section{Introduction}

The large sky surveys with a long-time base photometry gather copious amounts of light curves. Such missions as Kepler (Borucki et al. 2010), leave behind extensive catalogs of binaries, e.g. Kepler Eclipsing Binary Catalogue (KEBC, Prsa et al. 2011). One of the challenges in current astronomy is a proper and efficient classifications of light curves within such large databases. The classification of the intrinsic variability of the light curve has never been done before. Usually, in the case of close binaries, such variability is beeing explained as caused by the magnetic activity manifesting itself with starspots. Here we present a simple, yet effective, way to distinguish binaries with the presense of starspots and starspot activity.

Our method is based on the analysis of the evolution of the precise positions (in time and flux) of the light curve extrema. Below we present several starspot indicators. Based on those, we developed an algorithm that can be applicable to any long-time based photometric survey. All numerical simulations were performed using a modified version of the Wilson-Devinney code (Wilson \& Devinney 1971). Our model system parameters are based on the first contact binary we studied, KIC 2158793 (Debski et al. 2014). We tested the method on a sample of $422 \mathrm{EW}$-type light curves from KEBC. Here we present the results using the contact binary KIC 6118779, which is an exemplary system exhibiting a starspot migration (Debski et al. 2015). 

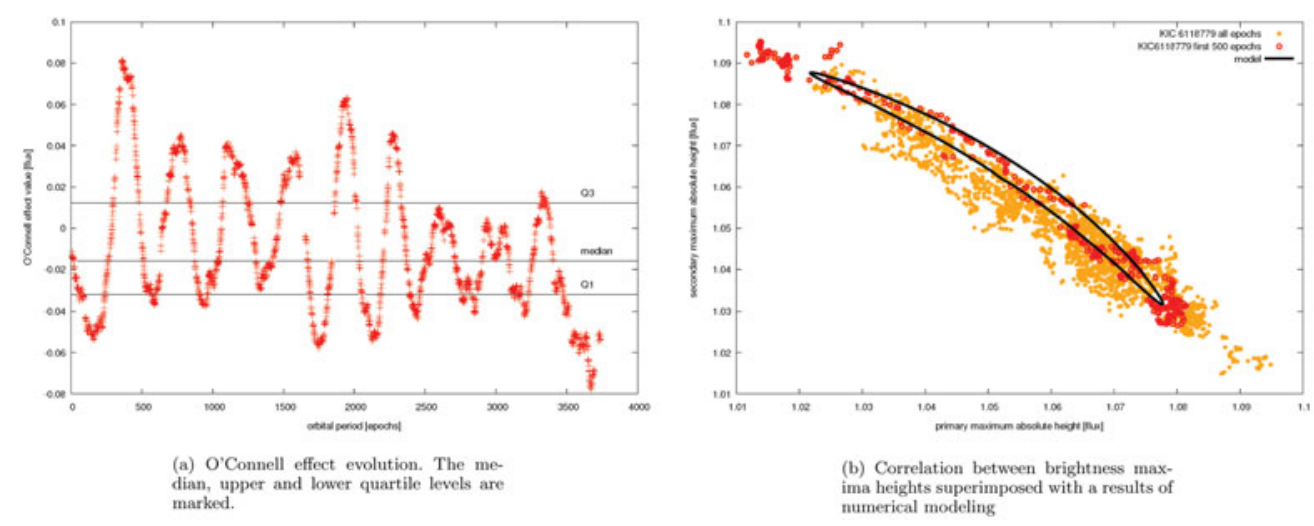

Figure 1. Light curve parameters evolution for KIC 6118779. Observations compared with a modeled evolution under the assumption of a migrating starspot is presented in panel b). First 500 epochs cover about one full migration of a starspot along the star's longitude. Please note slightly curved shape of the relation, in agreement with the model of starspot migration.

\section{O'Connell effect evolution}

The presence of a stationary, non-evolving star spot is in general manifested by the difference in the heights of the light curve maxima (the O'Connell effect, O'Connell 1951). Search for such systems can be made by the trivial detection of a non-zero amplitude of the O'Connell effect. In the case of systems with star spots changing with time, the mean O'Connell effect brings no valuable information. We study the way of changes of the O'Connell effect measuring its value in every subsequent orbital period. Since the unpredictable activity on a binary's surface may cause a single-time large-amplitude O'Connell effect, we chose to measure the median instead of the mean value to introduce the base level of the O'Connell effect for a whole span of observations of a given system. For consistency, we use interquartile range $\left(I Q R_{O^{\prime} \text { Connell }}\right)$ instead of standard deviation to describe the intensity of the evolution of the O'Connell effect amplitude.

As a star spot moves along the longitude on a contact binary, the light curve maxima change their heights, producing a characteristic signal in the evolution of the O'Connell effect amplitude. Contact binary KIC 6118779 is a great example of such a case (Fig. 1a). Detection of the signal can be made by measuring the regression coefficient of the relation between the primary and secondary maxima heights, $a_{O^{\prime} \text { Connell }}$ (see Fig. 1b). The longitudal starspot migration should result in anticorrelation between these parameters $\left(a_{O^{\prime} \text { Connell }}=-1\right.$, as seen in the Fig. $\left.1 b\right)$.

\section{Maxima separation}

As it turns out, there can be a starspot activity (varying in time) that will not cause an O'Connell effect. Such a stationary star spot must occur on the neck between the components of a binary or must be located on a stellar pole. In such a case no change in size nor temperature of the star spot will produce the O'Connell effect, however, the maxima still will change their position (in orbital phase). In the same time, the minima should stay not affected, therefore it could be possible to detect this type of activity by measuring the correlation between the maxima separation and the difference in the minima depths. For this case, the regression coefficient $a_{\text {Separation }}$ should be close to zero. In general, the maxima separation evolution can be treated as a firm indicator of the ongoing intrinsic changes of the light curve. That could be understood as the 


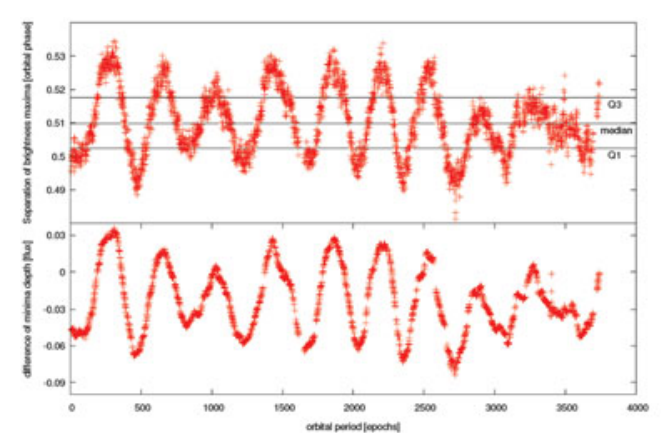

(a) Top: brightness maxima separation evolution; bottom: brightness minima depth difference evolution

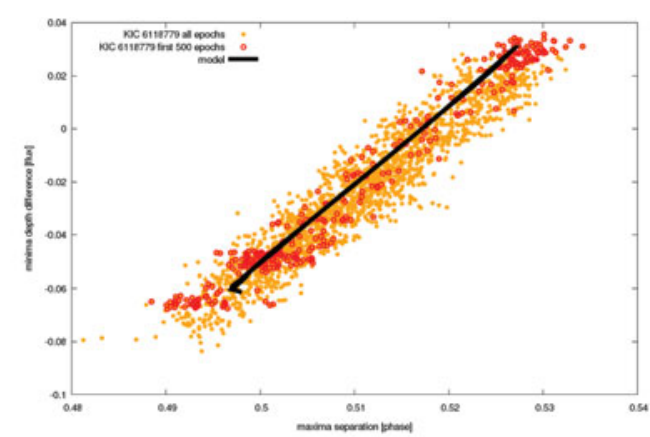

(b) Correlation between maxima separation and minima difference superimposed with a results of numerical modeling

Figure 2. Evolution of light curve parameters for KIC 6118779. Comparison with modeled evolution under the assumption of a migrating starspot is presented in panel b).

ongoing magnetic activity in the system. Therefore, we measure the amplitude of the maxima separation evolution with the interquartile range: $I Q R_{\text {Separation }}$. Any case of the evolving starspots will be detectable in the $I Q R_{\text {Separation. }}$. In case of a migrating starspot, in general $a_{\text {Separation }} \geqslant 1$, although the lower boundary for $a_{\text {Separation }}$ could possibly be decreased for low inclination systems.

\section{The algorithm}

We aim to provide a universal method of classification of intrinsically variable light curves of the presumably magnetically active close binaries. We present a simple algorithm that allowed us to sort and categorize our sample of 422 light curves from KEBC. The general flowchart presented in Fig. 3 is discussed in this section.

The first step was to download the detrended EW-type light curves from KEBC v2.0. As of new version of the catalog, light curve types are replaced by the morph parameter. In the newest attempt (not presented here) of classification we used all systems with morph $>0.6$, which proved to provide light curves with prominent maxima and minima. Unfortunately, using the morph parameter only let many non-EB/EW light curves to the analysis, returning no conclusive results. For that reason we decided to use the sample chosen from the first version of the KEBC. The second step is to recalculate the data from the Barycentric Julian Days (BJD) into the orbital phase domain. The zero-point here is the primary minimum for the epoch preceding the epoch of the first observation. Here we check, how many data points compose one orbital period to determine the binning window. In the next step we merge the phase curves into chunks using the binning window close to about 120 data points. We found this to be a good trade-off between the precision of the analysis and the timescale of the light curve evolution. Some very active binaries experience considerable intrinsic changes in a timescale of about 20 epochs, which imposed an upper boundary for the binning window. In the next three steps we perform search for the light curve parameters: we fit a high order polynomial to selected parts of the curve and store resulting extrema parameters in the form of time series. Then, after removing outlying data points, we calculate values of the indicators described in the previous two sections. At this point we have all pieces of information needed to classify the light curve.

The classification in respect to the IQRs is determined with the three threshold parameters: $\alpha, \beta$ and $\epsilon$, values depending on the precision of the photometry and the degree 


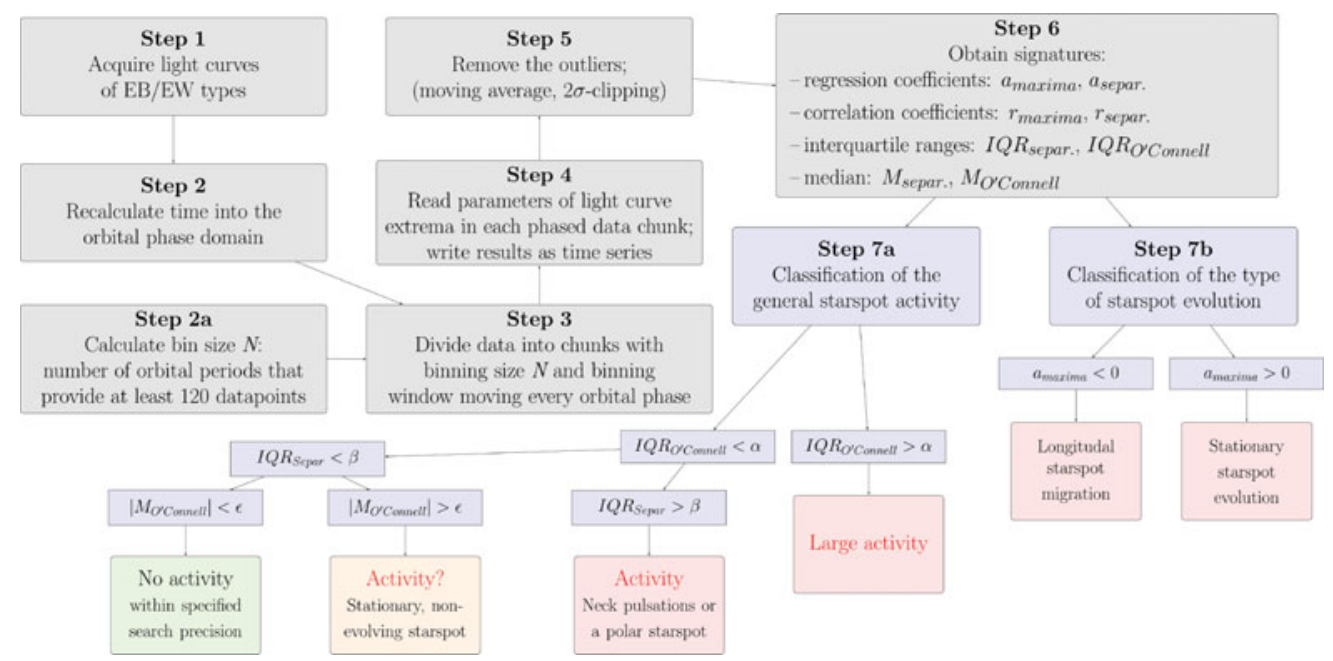

Figure 3. A flowchart of the algorithm

of accuracy a user desires to achieve. A somewhat different classification is presented in the branch of Step $7 b$. Here we search for signatures of a star spot migration. The $a_{\text {Separation }}$ indicator can be used as a confirmation that system should fall into the class of $I Q R_{O^{\prime} \text { Connell }}<\alpha$ and $I Q R_{\text {Separation }}>\beta$. The correlation coefficients can be used as auxiliary parameters for checking the reliability of $a_{\text {Separation }}$ and $a_{O^{\prime} \text { Connell }}$ values.

\section{Summary}

We have developed a new method that can be applied for classification of light curves of close binary systems taken by long-term photometric surveys. The main conclusions inferred from our analysis of the evolution of the shape of light curves are:

- Evolution of the O'Connell effect and maxima separation can be treated as indicators of migrating starspots

- It is possible to detect starspots that do not produce the O'Connell effect

- Presented method is fast and can be easily applicable to any long-term, photometric observations of close binaries

\section{Aknowledgements}

This study was partly funded by the NCN 2012/07/B/ST9/04432 grant.

\section{References}

Borucki, W. J., Koch, D., Basri, G. et al. 2010, Science, 977, 327

Debski, B., Baran, A., \& Zola, S. 2014, CoSka, 427, 43

Debski, B., Zola, S., \& Baran, A. 2015, ASPC, 293, 946

O'Connell, D. J. K.. 1951, Publ. Riverview Coll. Obs., 2, 85

Prsa, A., Batalha, N., Slawson, R. W., et al. 2011, AJ, 151, 83

Wilson, R. E. \& Devinney, E. J. 1971, ApJ, 166, 605 